Keywords: Combretastatin A4 Phosphate; rat; pancreatic head and hepatic tumours; diffusion kurtosis; dynamic T1 mapping; pixel-wise Tofts model

\title{
Vascular disrupting agent in pancreatic and hepatic tumour allografts: observations of location-dependent efficacy by MRI, microangiography and histomorphology
}

Ting Yin ${ }^{1}$, Yewei Liu ${ }^{1}$, Ronald Peeters ${ }^{2}$, Yuanbo Feng ${ }^{1,2}$, Jie Yu ${ }^{1,2}$, Uwe Himmelreich $^{3}$, Raymond Oyen ${ }^{2}$ and Yicheng $\mathrm{Ni}^{*, 1,2}$

${ }^{1}$ Theragnostic Laboratory, Department of Imaging and Pathology, Biomedical Sciences Group, KU Leuven, Herestraat 49, 3000 Leuven, Belgium; 'Department of Radiology, University Hospitals, KU Leuven, Herestraat 49, 3000 Leuven, Belgium and ${ }^{3}$ Division of Biomedical MRI, Department of Imaging and Pathology, Biomedical Sciences Group, KU Leuven, Herestraat 49, 3000 Leuven, Belgium

Background: Tumours growing in organs of different vascular environment could exhibit diverse responses to vascular disrupting agent (VDA). This study was aimed to identify in vivo imaging biomarkers for evaluation of pancreatic and hepatic tumours and comparison of their responses to a VDA Combretastatin A4 Phosphate (CA4P) using multiparametric MRI.

Methods: Male WAG/Rij rats were used for orthotopic pancreatic head tumour and hepatic tumour implantation; tumour growth was monitored by 3D isotropic MRI using a 3.0-T clinic scanner. Therapeutic intervention using CA4P was investigated by in vivo quantitative MRI measurements including T2/T1 relaxation mapping, diffusion kurtosis imaging and dynamic contrastenhancement (DCE) imaging. Animals were scarified $10 \mathrm{~h}$ after CA4P treatment for ex vivo validation using microangiography and histomorphology.

Results: State-of-the-art clinical MRI protocols were successfully adapted for imaging small animal tumour with high reliability. One hour after CA4P injection, marked vascular shutdown was detected with DCE MRI in both pancreatic and hepatic tumours. However, $10 \mathrm{~h}$ later, therapeutic necrosis was limited in pancreatic tumours compared with that in hepatic tumours $(P<0.01)$. Heterogeneous therapeutic changes were depicted in tumour lesions using pixel-wise Tofts model, which was generated from dynamic T1 mapping. In addition, tumour responses including haemorrhage, oedema and necrosis were detected using quantitative T2/T1 relaxation maps and diffusion kurtosis images, and were validated using histomorphology.

Conclusions: Using multiparametric imaging biomarkers, hepatic tumours were found to be significantly more responsive to CA4P than pancreatic tumours, which could be of reference for designing future clinical trials on this agent.

Combretastatin A4 Phosphate (CA4P), as one of the smallmolecule vascular disrupting agents (VDAs), targets abnormal endothelia of existing tumour blood vessels via tubulin binding, causing vascular shutdown and resultant massive tumour necrosis
(Tozer et al, 1999). However, after CA4P treatment, the tumour usually regrows from the remaining viable tumour tissue especially at the periphery (Dark et al, 1997; Salmon and Siemann, 2006). While being used in combination with other treatments such as

*Correspondence: Professor Y Ni; E-mail: yicheng.ni@kuleuven.be

Received 12 July 2017; revised 11 August 2017; accepted 23 August 2017; published online 14 September 2017

(C) 2017 Cancer Research UK. All rights reserved 0007-0920/17 
anti-angiogenic therapy, chemotherapy or radiotherapy, it shows promising results in preclinical studies (Siemann and Shi, 2008; Tozer et al, 2009; Wang et al, 2009; Li et al, 2011), and is currently under clinical trials for solid cancers (Rustin et al, 2010; Nathan et al, 2012; Chase et al, 2017).

Non-invasive MRI is useful to assess the therapeutic response of CA4P. The rapid tumour vascular disruption could be monitored by MRI as early as $30 \mathrm{~min}$ after CA4P administration (Thomas et al, 2006; Colliez et al, 2016). Dynamic-contrast enhanced (DCE) MRI is one of the most frequently used imaging approaches for tumour vascular evaluation. Although DCE is currently applied in over 100 early-phase clinical trials of antivascular therapies for different types of cancer (O'Connor et al, 2012), therapeutic effect of CA4P on pancreatic tumour is rarely studied with DCE MRI.

Previously, using a quantitative multiparametric MRI platform to characterise rat orthotopic pancreatic head tumour model on a clinical scanner (Yin et al, 2017a), DCE and histology revealed abundant vascular distribution within pancreatic tumours. In this study, we investigated therapeutic response to VDA by pancreatic tumour in rats. Furthermore, to mimic liver metastases from pancreatic cancer, which is a common feature in clinic, inter- and intra-individual comparisons were performed in animals bearing orthotopic pancreatic head and hepatic tumours from the same origin.

Apart from direct assessment of vascular permeability using DCE-MRI, the sensitivity of $\mathrm{T} 2 / \mathrm{T} 1$ relaxation and diffusion kurtosis parameters were investigated as well. Considering that therapeutic response might vary in different regions for each tumour, pixel-wise quantitative measurements were conducted.

In view of the intrinsic multiple blood supplies in the pancreas, we hypothesised that pancreatic tumours could react differently to the VDA, as compared with the tumours in other visceral organs, such as the liver, which exclusively own their hilar vessels. In this study, to verify our hypothesis, we have used multiparametric MRI to assess the tumour environment and to identify in vivo imaging biomarkers for evaluation and comparison of pancreatic and hepatic tumours and their responses to the therapy using CA4P.

\section{MATERIALS AND METHODS}

Animal model and experimental design. All experimental procedures were approved by the animal ethical committee in our institution. Eleven male WAG/Rij rats around $250 \mathrm{~g}$ were used for imaging study. Under systemic anaesthesia using intraperitoneal injection of sodium pentobarbital (Nembutal; Sanofi Sante Animal, Brussels, Belgium) at $40 \mathrm{mg} \mathrm{kg}^{-1}$, small cubes of rhabdomyosarcoma (R1) tumour harvested from donor animal were transplanted into the pancreatic head and/or left hepatic lobe, respectively (Wang et al, 2009; Li et al, 2013; Yin et al, 2017a). Tumour growth was monitored with 3D MRI 10 days after implantation. Therapeutic intervention began after one of the tumours could be clearly detected on diffusion-weighted images. CA4P of $20 \mathrm{mg} / \mathrm{kg}$ was given to animals via intravenous injection. Animals went through MRI scans before, 1 and $10 \mathrm{~h}$ after CA4P injection. Baseline MRI scans were performed the night before CA4P injection and on the day of CA4P intervention. Experimental flowchart is summarised in Figure 1A.

MRI acquisitions. Therapeutic effect of VDA CA4P was monitored using multiparametric MRI on a 3.0-T clinical MRI scanner (Magnetom Prisma, Siemens, Erlangen, Germany), with a 16-channel phase array wrist coil used as receiver. Animals were anaesthetised with isoflurane in air-oxygen mixture, and were monitored using the $1025 \mathrm{~T}$ module from SA instruments Inc. (Stony Brook, NY, USA) for respiration trigger. Animals were fasted for $4 \mathrm{~h}$ before imaging, and placed in supine position for scans.
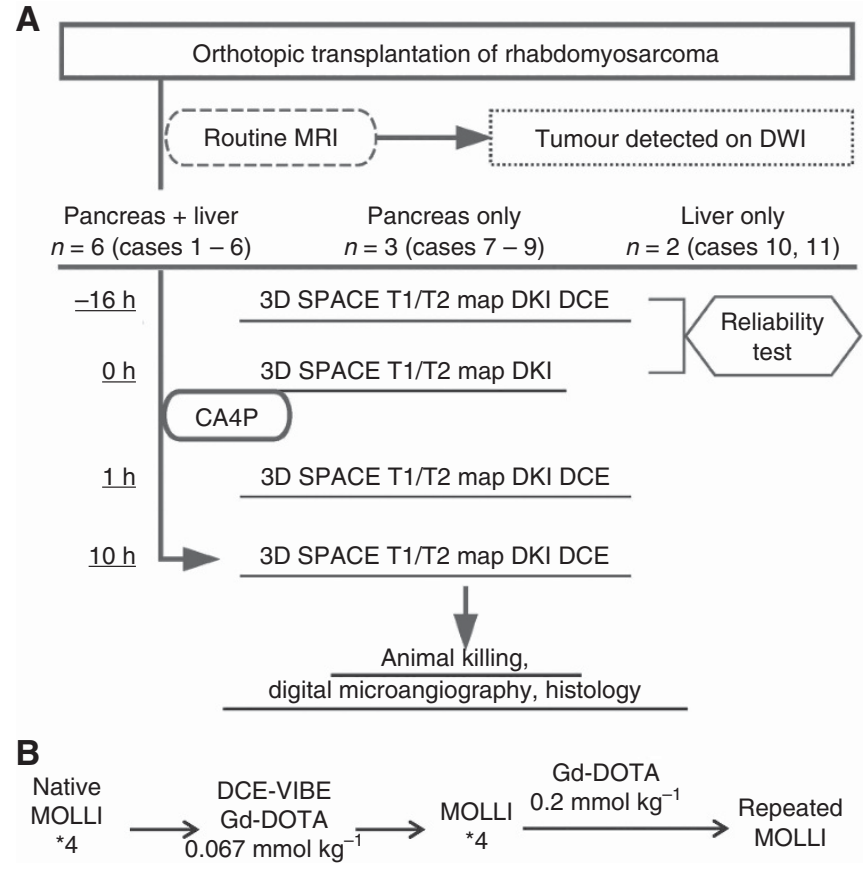

Figure 1. Flowchart of tumour therapeutic intervention study (A), and diagram of two dynamic contrast-enhancement protocols (B).

Abbreviations: $\mathrm{CA} 4 \mathrm{P}=\mathrm{Combretastatin} \mathrm{A} 4$ Phosphate; $\mathrm{DCE}=$ dynamic contrast-enhanced $\mathrm{MRI} ; \mathrm{DKI}=$ diffusion kurtosis imaging;

$D W I=$ diffusion-weighted imaging; $M O L L I=$ modified Look-Locker inversion recovery; SPACE $=3 \mathrm{D}$ turbo spin-echo with variable-flip angle refocusing $\mathrm{RF}$ pulses; $\mathrm{VIBE}=$ volumetric interpolated breath-hold examination.

All MRI protocols were optimised from standard clinical MR sequences as described previously, with a few adjustments in T2, T1 relaxation mapping and DCE protocols. MRI using a $0.39 \mathrm{~mm}$ $3 \mathrm{D}$ isotropic T2-weighted turbo spin-echo sequence (SPACE) was performed for anatomy reference and tumour volume estimation. Quantitative multiparametric measurements included 2D pixelwise T2, T1 relaxivity, apparent diffusion coefficient (ADC), apparent diffusion kurtosis (Kapp) mapping, region of interest (ROI)-based semiquantitative and pixel-wise quantitative DCE analysis. For 2D protocols, single-slice scans were applied to the largest section of the tumour. In case both pancreatic and hepatic tumours clearly presented in the same animal, but not on the same $2 \mathrm{D}$ slice, images of tumours were acquired from two sequential slices, with priority given to the pancreatic tumour. MRI protocols were performed twice before CA4P injection for reliability test, and repeated at 1 and $10 \mathrm{~h}$ after $\mathrm{CA} 4 \mathrm{P}$ intervention, as described in the flowchart (Figure 1A).

In order to minimise random motion artefact raised from the abdominal region, scanning duration for a single measurement in 2D images was further accelerated. To be specific, 24-echo T2 mapping was acquired from two repeated measurements instead of two averages, with each measurement finished within $150 \mathrm{~s}$; conventional inversion recovery turbo-flash (TFL) T1 mapping was acquired using nine inversion times (IR) from 168 to $2500 \mathrm{~ms}$, and each measurement was accomplished around 9-13 s. Diffusion kurtosis imaging (DKI) was achieved through 10 -b values varying from 0 to $2500 \mathrm{~s} \mathrm{~mm}^{-2}$ for both ADC and Kapp estimations.

DCE was performed using two different strategies: conventional DCE-VIBE (volumetric interpolated breath-hold examination) protocol with low-dose Gd-DOTA (Dotarem, Guerbet, France) was applied for all the animals, and dynamic modified LookLocker inversion recovery (MOLLI) mapping after high-dose Gd-DOTA injection was performed in eight animals (Cases 1-6, 9 
A

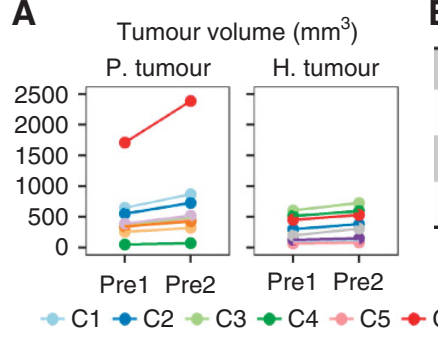

B

\begin{tabular}{lc|c|c|c|c|c|c|c}
\hline & \multicolumn{3}{c}{ Coefficient of variation (\%) of each MRI measurement } \\
\hline & \multicolumn{3}{c}{ Pancreatic tumour } & \multicolumn{3}{c}{ Hepatic tumour } \\
& T2 map & T1 map & ADC & Kapp & T2 map & T1 map & ADC & Kapp \\
\hline CVintra & 16.74 & 11.46 & 7.93 & 10.96 & 5.45 & 3.06 & 7.18 & 19.47 \\
\hline CVinter & 1.27 & 5.42 & 6.79 & 7.07 & 1.87 & 2.75 & 8.03 & 9.35 \\
\hline
\end{tabular}

\section{T2-weighted 3D SPACE}
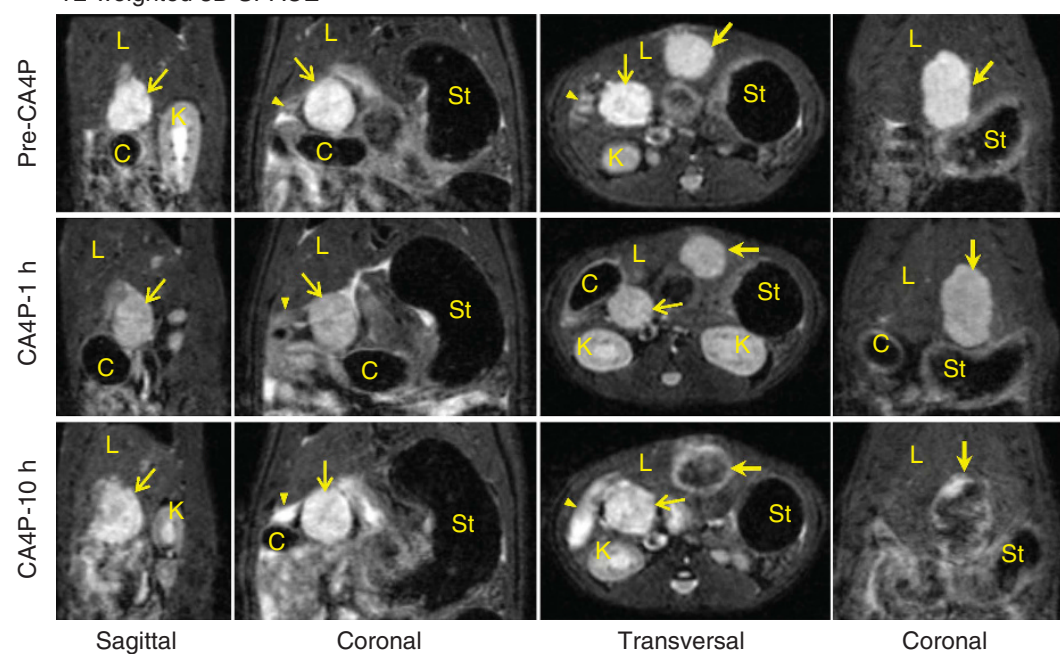

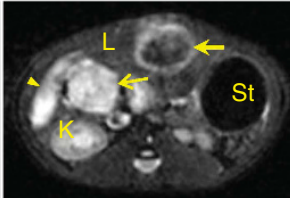

Transversal

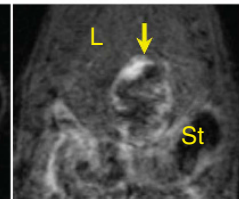

Coronal

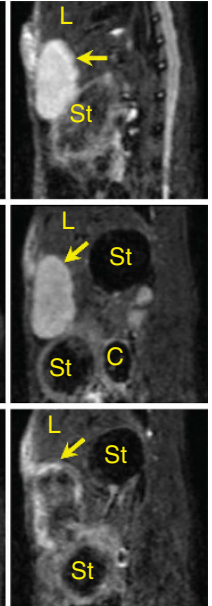

Sagittal

Figure 2. Baseline tumour growth, MRI reliability measurements and visual tumour response after CA4P therapy. (A) After an overnight growth, tumour volume increased $\sim 28.2 \%$ upon CA4P intervention (Pre2) compared with the volume obtained 16 h before CA4P injection (Pre1). (B) Low intersubject coefficient of variation (CVinter) indicated that T2/T1 relaxation and DKI measurements were not affected by overnight tumour growth, whereas higher variation was noticed from intrasubject measurements. (C) Both pancreatic tumour (thin arrow) and hepatic tumour (thick arrow) were clearly identified from T2-weighted 3D SPACE images, as hyperintense mass before CA4P treatment. Hypointense haemorrhagic necrosis was detected in hepatic tumour after $10 \mathrm{~h}$ of CA4P injection. Images from Case 4 were shown as an example. C 1-C 11: individual colour code for Case 1-Case 11. Abbreviations: C, colon; L, liver; K, kidney; St, stomach. Triangle arrow head, duodenum; thin arrow, pancreatic tumour; thick arrow, hepatic tumour.

and 11). More specifically, DCE-VIBE images were collected using free-breathing flash sequence with Siemens inline motion correction at a temporal resolution of $4.1 \mathrm{~s}$. After 15 baseline acquisitions, Gd-DOTA at $0.067 \mathrm{mmol} \mathrm{kg}^{-1}$ was injected via tail vein, followed by 65 post-contrast scans. In addition, $8 \mathrm{~min}$ after the first contrast injection, Gd-DOTA at $0.2 \mathrm{mmol} \mathrm{kg}^{-1}$ was injected for pixel-wise DCE analysis (Figure 1B); dynamic T1 maps were acquired using repeated MOLLI sequence with 5(2)3(4) scheme, which is an adaption from the standard cardiac 5(3 s) 3 scheme as described previously (Kellman et al, 2012). In this study, MOLLI T1 mapping was triggered by animal respiration signal. Five images were acquired after the first inversion during five breaths (first readout at $147 \mathrm{~ms}$ ), followed by two breath pauses, another three images were acquired after the second inversion in three breaths (first readout at $227 \mathrm{~ms}$ ), and the next MOLLI measurement started after four breath pauses. In this way, in total of eight IR images were acquired in 14 breaths (around $20 \mathrm{~s}$ ) for a single T1 mapping, and there are always seven breaths' interval between inversions throughout the entire dynamic T1 measurements. In addition, for animals undergoing DCE-MOLLI scans, DCE-VIBE experiments were carried out using $0.2 \mathrm{mmol} \mathrm{kg}^{-1}$ of Gd-DOTA $2-3$ days before tumour implantation, especially for acquiring population vascular input function (VIF). Detailed MRI parameters are summarised in Supplementary Table 1.

Image processing. For tumour volume estimation, tumours were semi-automatically segmented from 3D SPACE images using ITKSNAP software (Yushkevich et al, 2006). Pixel-wise MRI relaxation time mapping, ADC, Kapp and ROI/pixel-wise DCE data were processed using in-house built programmes written in Matlab (2016a, MathWorks, Natick, MA, USA), as previously described (Yin et al, 2017a). Averaged T2 relaxation map was generated from two separate measurements after non-rigid registration. Dynamic T1 MOLLI was processed with standard three-parameter fitting (Kellman et al, 2012).

For ROI-based DCE-VIBE data, a freehand ROI was placed on the tumour and tissue concentration time curve (CTC) was converted from corresponding VIBE signal. Semiquantitative parameters including initial area under curve $(90 \mathrm{~s})$, peak enhancement, wash-in and wash-out slope were measured from CTC in DCE-VIBE. In the meantime, pixel-wise CTC after highdose Gd-DOTA was directly extracted from dynamic MOLLI analysis. Combining with population VIF information obtained from the inferior vena cava in earlier experiments, perfusion kinetic parameters were obtained from pixel-wise CTC using standard Tofts model.

Postmortem digital microangiography and histology analysis. Immediately after the last MRI scans, animals were killed for postmortem verification. The pancreas was locally infused with Evans Blue dye for pancreas separation (Yin et al, 2017b), followed by barium sulphate suspension (Micropaque, Guerbet, France) injected via the abdominal aorta. The entire specimen of tumourbearing pancreas and/or liver were dissected for digital microangiography (Chen et al, 2006), which was performed with a clinical mammographic unit (Mammomat Inspiration, Siemens, 


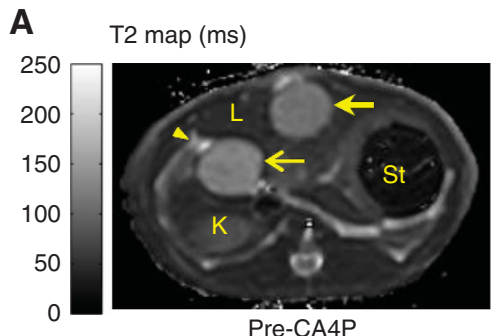

T2 value (ms): pancreatic tumour

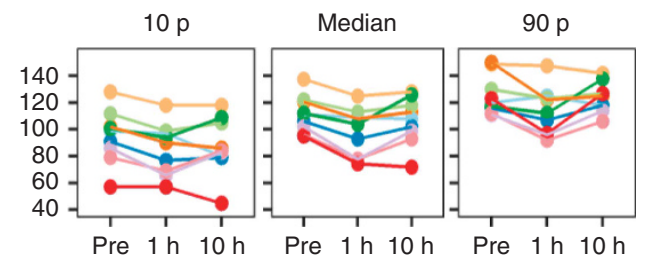

B T1 map (ms)

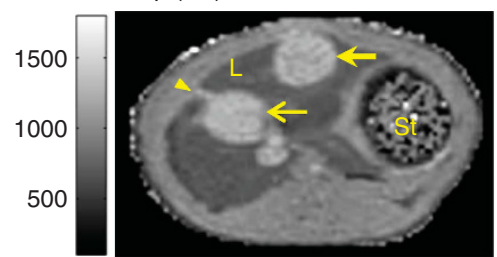

Pre-CA4P
CA4P-1 h

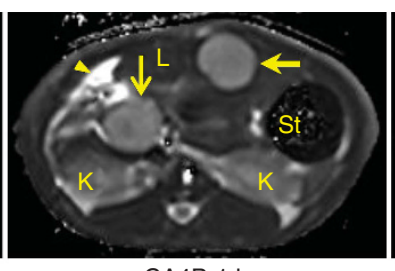

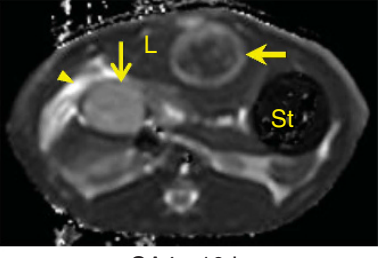

CA4p-10 h

Hepatic tumour

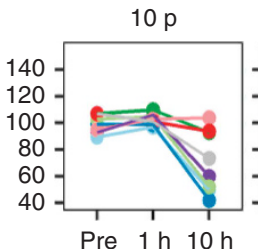

Median

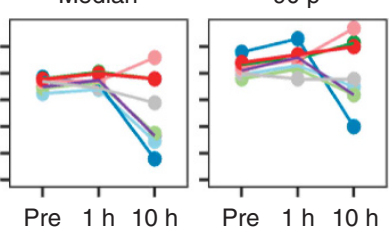

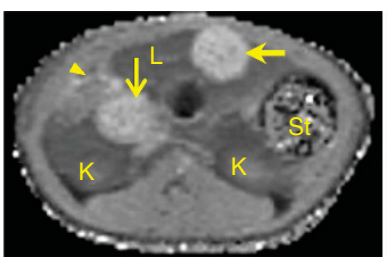

CA4P-1 h

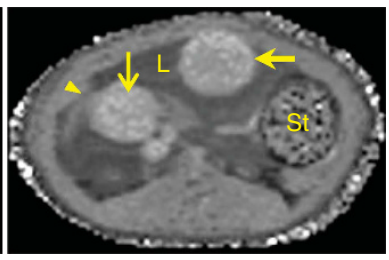

CA4P-10 h

T1 value (ms): pancreatic tumour

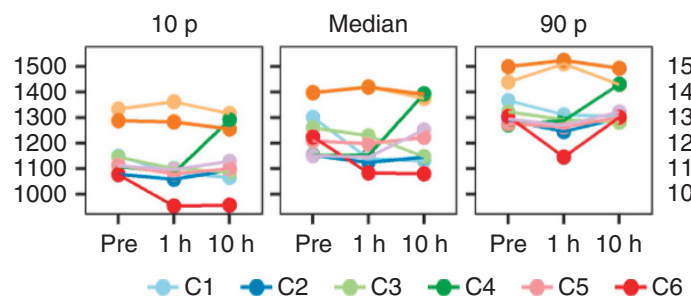

Hepatic tumour

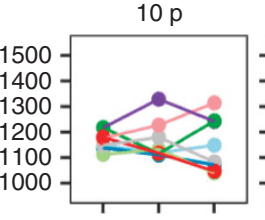

Median

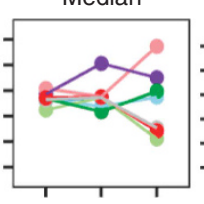

Pre $1 \mathrm{~h} 10 \mathrm{~h}$ Pre $1 \mathrm{~h} 10 \mathrm{~h}$ Pre $1 \mathrm{~h} 10 \mathrm{~h}$

$90 \mathrm{p}$

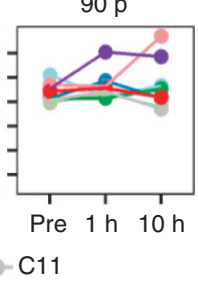

Figure 3. Relaxation maps for pancreatic and hepatic tumours, images from Case 4. (A) After $1 \mathrm{~h}$ of CA4P treatment, severe vascular collapsing resulted in a decrease of T2 values $(P<0.01)$ in pancreatic tumours (thin arrow); pancreatic T2 value returned to baseline after $10 \mathrm{~h}$ of therapy. Haemorrhagic necrosis dominated in hepatic tumours (thick arrow) gradually after CA4P intervention, with the presence of viable tumour rim. (B) Native T1 maps indicate diverse patterns after treatment. Abbreviations: L, liver; K, kidney; St, stomach. Arrow head, duodenum; thin arrow, pancreatic tumour; thick arrow, hepatic tumour.

Germany), exposed at $28 \mathrm{kV}$ and $18 \mathrm{mAs}$. Afterwards, tumourbearing organs were fixed in $10 \%$ formalin and paraffin-sectioned for haematoxylin-eosin (HE) staining and microscopy.

Statistical analysis. Tumour ROI was manually delineated for histogram analysis in R software (v3.1.1, https://cran.r-project.org), for the reporting of the 10th, 50th and 90th percentile. Coefficient of variation $(\mathrm{CV})$ calculated as the s.d. divided by the mean value was applied as an index of MRI reliability. Two-way ANOVA was used for comparisons of quantitative MRI data, and Tukey's HSD was applied for post hoc testing. Differences with a $P$-value lower than 0.05 were considered statistically significant.

\section{RESULTS}

Tumour growth and baseline parametric MRI reliability. By the time planned for CA4P investigation, in total of 17 tumour grafts could be unambiguously detected on multiparametric MRI. Results were documented for each individual animal, coded as Case 1 to Case 11 (abbreviation C 1-C 11). To be specific, C 1-6 with both pancreatic head tumour and hepatic tumour, $\mathrm{C}$ 7-9 with only pancreatic tumour and C 10 and C 11 with only hepatic tumour were included for imaging study on CA4P therapeutic efficacy (Figure 1A). Minor intratumoural haemorrhage was observed in pancreatic tumours of $\mathrm{C} 1,2,5,8$ and 9, whereas major intratumoural haemorrhage and spontaneous necrosis were noticed in pancreatic tumour of Case 6. As for the hepatic tumours, the tumour homogeneity was much higher, and neither intratumoural haemorrhage nor necrosis was detected during baseline MRI in all cases.

Tumour volume obtained from T2-weighted 3D SPACE images was summarised in Figure 2A. In general, tumour volume increased for $\sim 28.2 \%$ on the day of CA4P intervention, with median tumour size around $499 \mathrm{~mm}^{3}$ (range $74-2388 \mathrm{~mm}^{3}$ ) in the pancreas and $350 \mathrm{~mm}^{3}$ (range $87-720 \mathrm{~mm}^{3}$ ) in the liver lobe. Baseline T2/T1 relaxation and diffusion kurtosis measurements were not affected by tumour growth, with intersubject CV (CVinter) under 10\%, as shown in Figure 2B. However, because of the large range of baseline tumour size, intrasubject $\mathrm{CV}$ (CVintra) was higher than $10 \%$, in general within $20 \%$.

Visual inspection of tumours on T2-weighted 3D MRI. Upon CA4P intervention, all tumours included in imaging study were clearly identified on T2-weighted 3D SPACE with hyperintense signal (Figure 2C). Pancreatic head tumour located inferior to the 
A $\quad$ ADC map (mono-exponential, $10^{-3} \mathrm{~mm}^{2} \mathrm{~s}^{-1}$ )

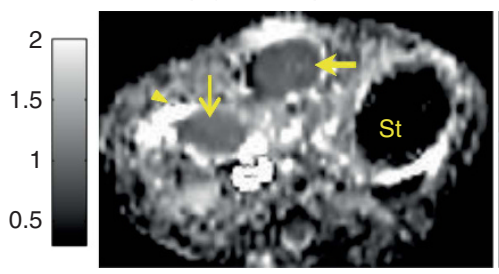

Pre-CA4P

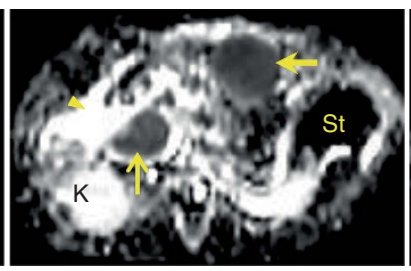

CA4P-1 h

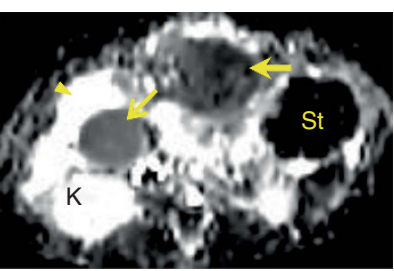

CA4P-10 h

ADC value $\left(10^{-3} \mathrm{~mm}^{2} \mathrm{~s}^{-1}\right)$ :

Pancreatic tumour
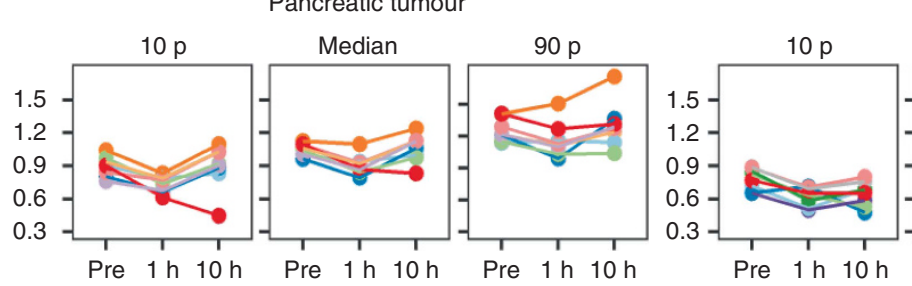

Hepatic tumour

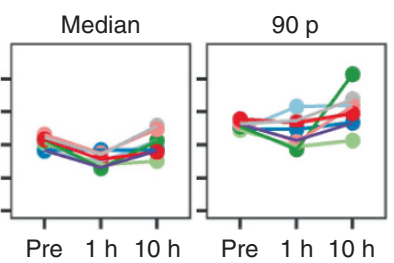

B DKI kurtosis map

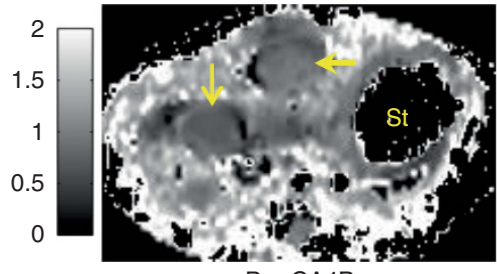

Pre-CA4P

DKI Kurtosis: pancreatic tumour

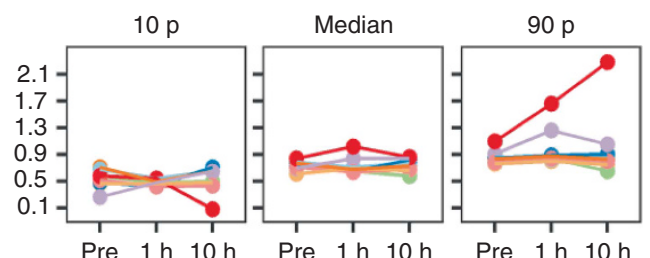

$-\mathrm{C}_{1}-\mathrm{C} 2-\mathrm{C}_{3}-\mathrm{C} 4-\mathrm{C} 5-\mathrm{C} 6$

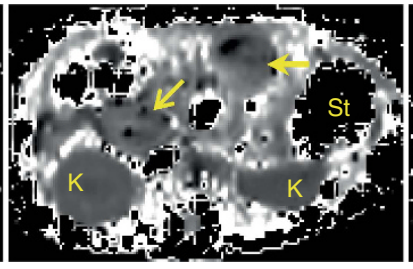

CA4P-1 h

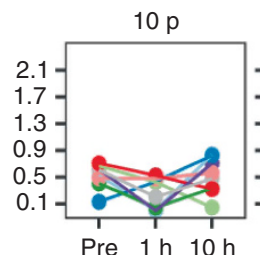

Pre $1 \mathrm{~h} 10 \mathrm{~h}$

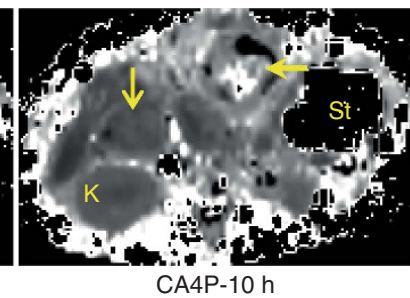

Hepatic tumour

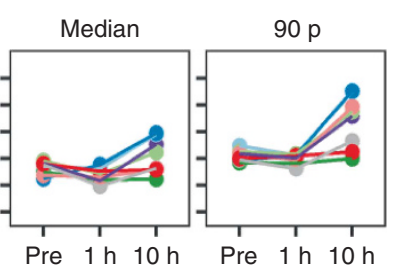

Figure 4. Diffusion kurtosis imaging of tumour therapy, images from Case 4. (A) Haemorrhage led to a decrease in ADC values in both pancreatic (thin arrow) and hepatic tumours (thick arrow) $1 \mathrm{~h}$ after CA4P injection $(P<0.01)$. Further increased ADC values in hepatic tumours indicated the generation of necrosis core $(P<0.01,1 \mathrm{vs10h})$. (B) Diffusion kurtosis rose up in case of continued haemorrhage and clot formation $(P<0.01)$. Abbreviations: K, kidney; St, stomach. Arrow head, duodenum; thin arrow, pancreatic tumour; thick arrow, hepatic tumour.

right liver lobe and anterior to the right kidney, closely wrapped by colon and duodenum loops, whereas hepatic tumour was located in the left liver lobe, adjacent to the stomach. Visually, tumour morphology did not change $1 \mathrm{~h}$ after CA4P injection. Ten hours after CA4P injection, hypointense haemorrhagic necrosis core was detected in all hepatic tumours. However, no apparent response is shown in pancreatic tumour, except for $\mathrm{C} 6$, in which tumour necrosis further expanded.

$\mathrm{T} 2 / \mathrm{T} 1$ relaxation and diffusion kurtosis measurements. Compared with native $\mathrm{T} 1$ relaxation measurement, T2 mapping showed higher sensitivity for intratumoural haemorrhage induced by CA4P vascular disruption (Figure 3). Meanwhile, necrosis was reflected on DKI (Figure 4). In general, pancreatic and hepatic tumours showed very different responses to CA4P intervention. For pancreatic tumours (excluding C6), both T2 (Figure 3A) and ADC (Figure 4A) values decreased $1 \mathrm{~h}$ after CA4P injection because of acute haemorrhage $(P<0.01)$, and gradually recovered $10 \mathrm{~h}$ after treatment, while native $\mathrm{T} 1$ (Figure $3 \mathrm{~B}$ ) and Kapp (Figure 4B) values did not indicate apparent patterns. For pancreatic tumour in $\mathrm{C} 6$, necrosis region propagated throughout the entire tumour after therapeutic intervention, resulting in a decreased $\mathrm{T} 2, \mathrm{~T} 1$ relaxation and $\mathrm{ADC}$ values, together with a raised 90th percentile in Kapp histogram (Figure 4B). On the contrary, acute haemorrhagic necrosis was identified in all hepatic tumours, as indicated by increased ADC and Kapp values (Figures 4, $P<0.01$ ). Meanwhile, T2 values further decreased within the intratumoural haemorrhagic necrosis, whereas increased in the non-haemorrhage region (Figure 3A). Different combinations of haemorrhage and necrosis contents also resulted in diverse T2 histogram. The DKI data for the pancreatic tumour in C 4 were excluded because of the insufficient tumour size.

DCE MRI. Population vascular input function (VIF) measured with DCE-VIBE sequence combined with $0.2 \mathrm{mmol} \mathrm{kg}^{-1}$ of GdDOTA was fitted into a bi-exponential function: $\mathrm{Cp}(t)=D \times$ $(\mathrm{a} 1 \times \exp (-\mathrm{m} 1 \times t)+\mathrm{a} 2 \times \exp (-\mathrm{m} 2 \times t))$, where administered dose $D=0.2 \mathrm{mmol} \mathrm{kg}^{-1}$, amplitude a1 $=6.9854 \mathrm{~kg} \mathrm{mmol}^{-1}$, $\mathrm{a} 2=5.9849 \mathrm{~kg} \mathrm{mmol}^{-1}$, and decay constants $\mathrm{ml}=0.81999$ $\min ^{-1}, \mathrm{~m} 2=0.036687 \mathrm{~min}^{-1}$. Pharmacokinetic (PK) parameters were obtained from standard Tofts model using estimated population VIF.

DCE experiments provided direct information for CA4Pinduced vascular disruption and tumour revascularisation, as 
A DCE-VIBE (ROI analysis)

P. tumour H. tumour

iAUC (mM.min)

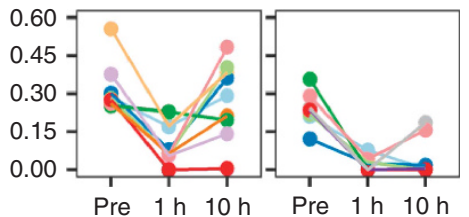

Pre $1 \mathrm{~h} 10 \mathrm{~h}$

Peak enhance (mM)

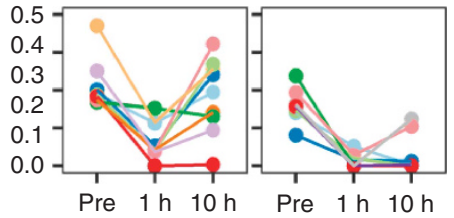

Wash-in $\left(\mathrm{mM} \mathrm{min}^{-1}\right)$

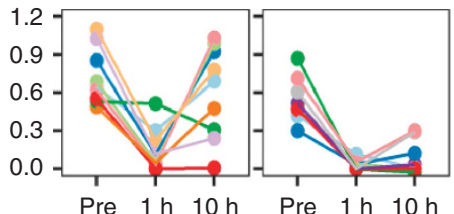

Wash-out $\left(\mathrm{mM} \mathrm{min}^{-1}\right)$

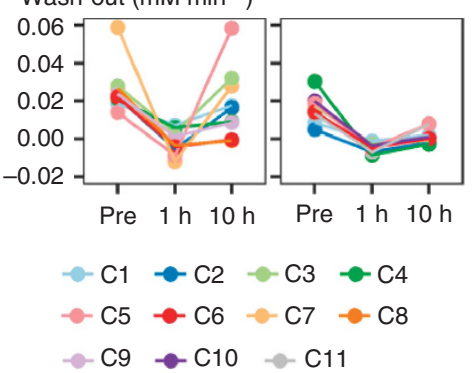

B DCE-MOLLI (pixel-wise Tofts model) Ktrans map $\left(\mathrm{min}^{-1}\right)$

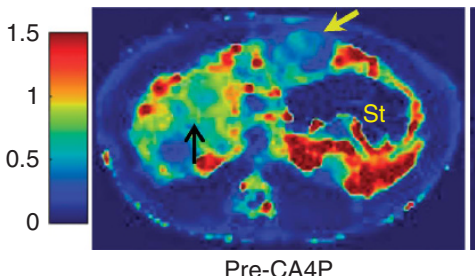

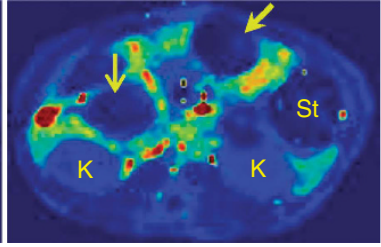

CA4P-1 h

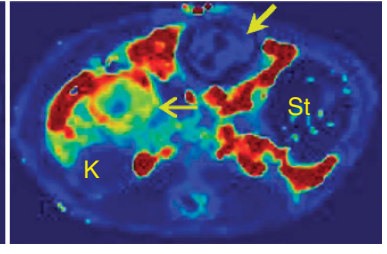

CA4P-10 h

Ktrans $\left(\mathrm{min}^{-1}\right)$ : pancreatic tumour
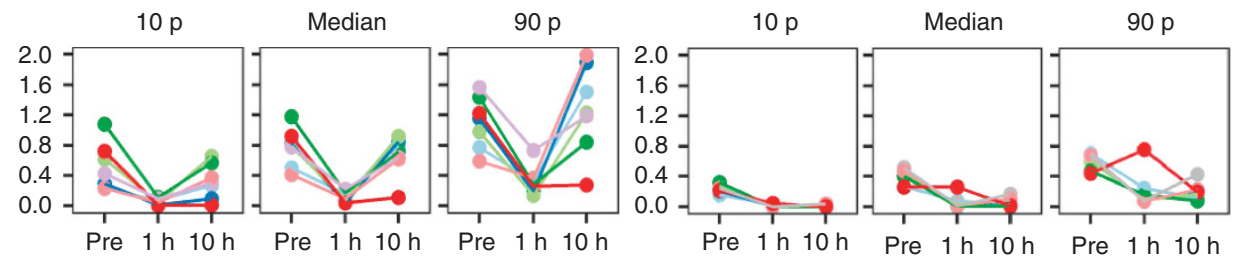

C

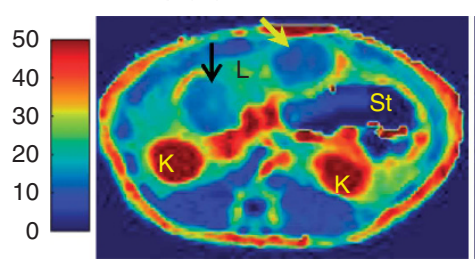

Pre-CA4P ve (\%): pancreatic tumour $10 p$

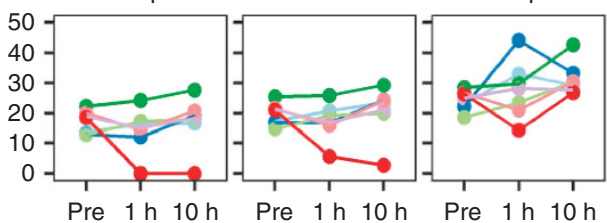

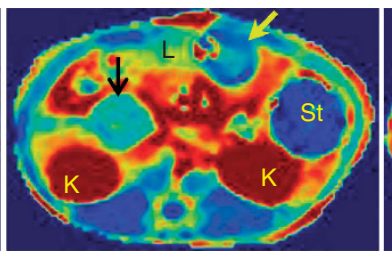

CA4P-1 h

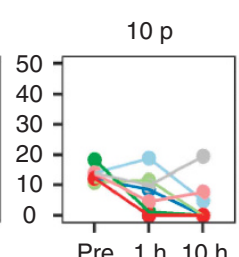

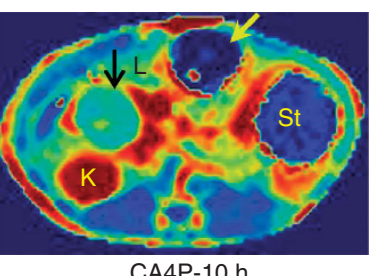

Hepatic tumour Median

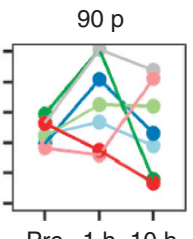

Figure 5. DCE monitoring of CA4P response (derived from Case 4). (A, B) Vascular disruption was clearly revealed from DCE parameters, with decreased initial uptake, peak enhancement, wash-in, wash-out and kinetic Ktrans values at $1 \mathrm{~h}(P<0.01$ vs baseline). Ten hours after CA4P injection, tumour re-perfusion was much more obvious in pancreatic tumours than that in hepatic tumours $(P<0.01)$. (C) Because of the mixture of clot and acute necrosis, extracellular volume fraction ve presented a randomised or heterogeneous pattern. Abbreviations: $L$, liver; $K$, kidney; St, stomach. Thin arrow, pancreatic tumour; thick arrow, hepatic tumour.

shown in Figure 5. Before therapeutic intervention, both pancreatic tumour and hepatic tumour exhibited typical type III curve pattern, with rapid contrast wash-in followed by fast wash-out. Tumour perfusion in pancreatic tumours was generally greater than perfusion in hepatic tumours $(P<0.01)$, as indicated from semiquantitative parameters iAUC, peak enhancement, wash-in slope (Figure 5A) and pixel-wise quantitative PK parameter Ktrans map (Figure 5B and Supplementary Table 2). Vascular shutdown was observed in all tumours $1 \mathrm{~h}$ after CA4P injection, as reflected in both semiquantitative data and Ktrans maps. The negative wash-out slope during this phase indicated a transition from type III curve to progressive type I curve (continuously slow wash-in). Ten hours after CA4P treatment, because of fast vascular reperfusion in pancreatic tumours, all tumours were spontaneously re-perfused except in C 6. Meanwhile, pancreatic tumour exhibited a heterogeneous response to $\mathrm{CA} 4 \mathrm{P}$, and increased 90th percentile of extracellular volume (ve) indicated the presence of small oedematous necrosis fraction in pancreatic tumour (Figure 5C). On the contrary, tumour vasculature did not recover in most part of hepatic tumours. Besides, because of haemorrhage, Gd-DOTA access to extracellular space was hampered in the area with clot, resulting in a decreased 10th percentile of ve in hepatic tumours (Figure 5C).
Postmortem findings. Tumour vascularisation was confirmed by ex vivo microangiography and HE staining, as shown in Figure 6. Corresponding digital photos for gross specimens and microangiography were shown in Figure 6A and B. Barium sulphate-perfused pancreatic tumour presented prominent hyperdense signal, implying tumour vessel re-perfusion $10 \mathrm{~h}$ after CA4P treatment (Figure 6B). However, hypodense signal surrounded by peripheral hyperdense vessels in hepatic tumour indicated a necrotic core surrounded by a rim of viable tumour tissue (Figure 6B). Minimum necrosis fraction was detected on HE-stained pancreatic tumour slices, with residue haemorrhage (Figure 6C and D). By contrast, massive damage, haemorrhage and necrosis with minimum proportion of viable tumour tissue mainly at the periphery were observed on hepatic tumour slices (Figure 6C and D).

\section{DISCUSSION}

CA4P is currently widely used in clinical trails as a VDA for solid cancer therapy. However, tumours growing in organs of different vascular environments could exhibit diverse responses to VDAs ( $\mathrm{Li}$ et al, 2013). Compared with other organs, pancreas, especially the pancreatic head portion has abundant and complicated vascular 
A

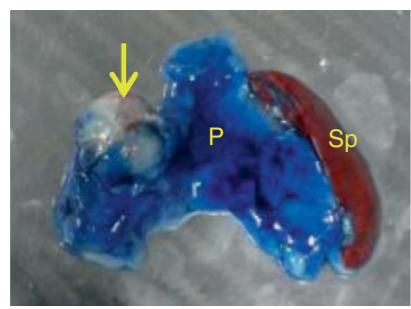

B

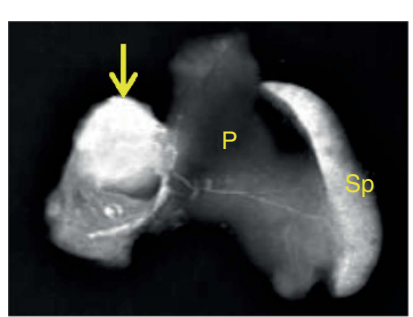

C

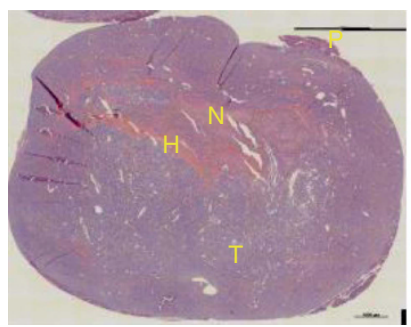

D

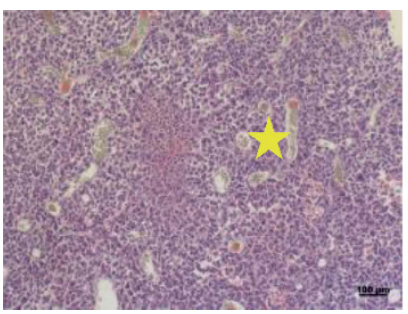

Hepatic tumour
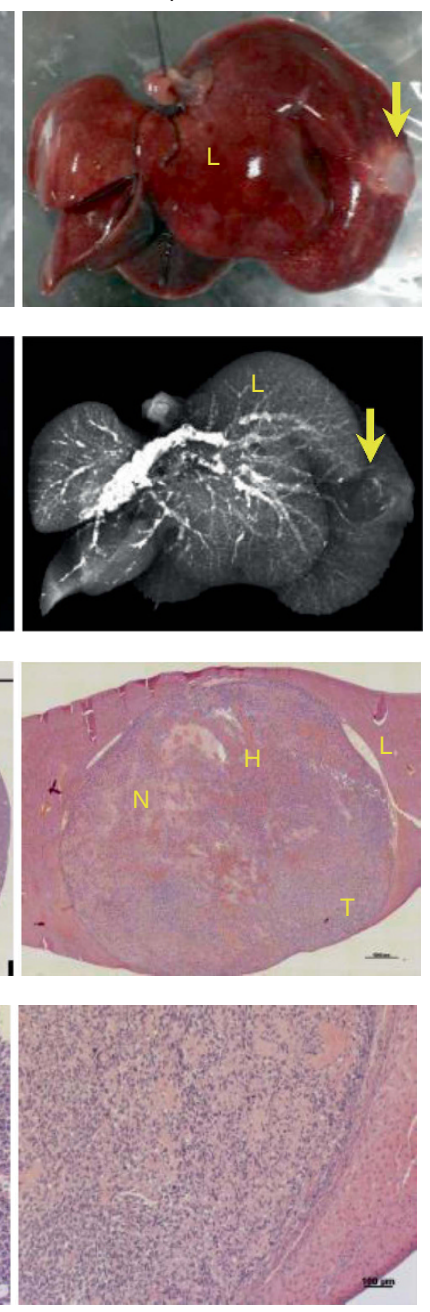

Figure 6. Results of ex vivo microangiography and histology.

(A) Digital photos of pancreatic and hepatic tumours imbedded in the pancreas and liver lobe, respectively, with the pancreas infused with Evans Blue. (B) Microangiography indicated much more abundant vasculature in pancreatic tumour $10 \mathrm{~h}$ after CA4P therapy, compared with diminished blood supply in hepatic tumour. (C) HE-stained gross slices clearly presented the diverse proportions of haemorrhagic necrosis in pancreatic and hepatic tumours, scale bar: $1 \mathrm{~mm}$. (D) Microscopic images confirmed the different tissue viability and vascular distribution within pancreatic and hepatic tumours, scale bar: $100 \mu \mathrm{m}$. Abbreviations: $\mathrm{H}$, haemorrhage; $L$, liver; $N$, necrosis; $P$, pancreas; $S p$, spleen; $T$, tumour. Star, vessel; thin arrow, pancreatic tumour; thick arrow, hepatic tumour.

supplies (Okahara et al, 2010), which may cause unexpected VDA therapeutic outcomes. Indeed, inter- and intra-individual comparisons in this study demonstrated that tumours of the same origin responded differently to the same VDA treatment. Unlike liver tumours that were all profoundly treated by CA4P, pancreatic head tumours rapidly recovered from the drug insult, most likely because of sufficient blood flows from the surrounding supplying vessels to this unique organ that lacks the hilum, a structure common to all other visceral organs. Therefore, other alternatives such as surgical resection, radiofrequency ablation, microwave, absolute ethanol, and so on might be necessary to treat the tumours located in the pancreas when a VDA is considered to treat metastatic tumours elsewhere.

In tumour therapeutic studies using CA4P, DCE MRI could provide direct information about tumour vascularity, whereas other tumour responses including haemorrhage, oedema and

necrosis could also be monitored in combination with other multiparametric MRI protocols. The present work provides a preclinical MRI imaging research platform to assess tumour responses to $\mathrm{CA} 4 \mathrm{P}$ treatment with high reliability and flexibility. State-of-the-art clinical MRI protocols were translated and further optimised for small animal research.

To avoid spontaneous intratumoural haemorrhage, therapeutic intervention was planned while one of the tumours (pancreatic or hepatic) reached around $500 \mathrm{~mm}^{3}$. However, under such circumstances, the volume of the other tumour might be relatively too small (below $50 \mathrm{~mm}^{3}$ ), and difficult to be detected with MRI. In this study, pixel-wise T2/T1 relaxation mapping and DCE-MOLLI measurements were robust for the assessment of small tumours around $100 \mathrm{~mm}^{3}$.

Generally speaking, because of the complex anatomy, MRI characterisation of pancreatic tumours is far more challenging than imaging hepatic tumours in rats. Random motion artefact from small bowel and colon could impair image quality. Therefore, only one 2D slice with the largest section was scanned for each tumour, and 2D imaging protocols were further accelerated in each measurement. However, poor field homogeneity surrounding the pancreatic tumour induced severe distortion in DWI, resulting in failure of quantification for the small pancreatic tumour (Case $4,74 \mathrm{~mm}^{3}$ ).

DCE MRI is one of the most widely used techniques to investigate effects of VDAs, in both preclinical phase and clinical trials (Nielsen et al, 2012; Fruytier et al, 2014; Colliez et al, 2016). Here in this study, two DCE protocols were combined to provide semiquantitative information and haemodynamic parameters. DCE-VIBE imaging with high temporal resolution (4.1 s) revealed more precise information about peak enhancement within the tumour. However, because of the low spatial resolution and disruptive motion artefact, DCE-VIBE could only provide ROI-based information for the entire tumour. Meanwhile, we expected a heterogeneous response within each tumour; thus, pixel-wise dynamic MOLLI T1 mapping was dedicated for kinetic modelling with high spatial resolution. Repeated MOLLI T1 mapping was previously introduced for cardiac extracellular volume evaluation (Kellman et al, 2012), where the change in myocardial $\mathrm{T} 1$ was measured following contrast administration. Here, dynamic MOLLI T1 mapping was further modified as an alternative to compensate traditional DCE measurements to achieve pixel-wise analysis.

There are a few limits for this modified DCE-MOLLI protocol. Because of the relative low temporal resolution in each MOLLI measurement (20s for each T1 map), it might not be able to capture the real peak enhancement; on the other hand, kinetic modelling after curve fitting showed higher tolerance to dynamic MOLLI data. According to previous report, with such temporal resolution, Ktrans might be underestimated for $\sim 4 \%$, and ve being overestimated $\sim 1 \%$ (Heisen et al, 2010). As tumour response to CA4P was marked, error around $1-4 \%$ is still acceptable.

It is important to have a reliable VIF for kinetic modelling for DCE acquisition. However, it is not realistic to obtain VIF from single slice MOLLI measurement, also difficult to measure VIF from DCE-VIBE technique with $0.067 \mathrm{mmol} \mathrm{kg}^{-1}$ of Gd-DOTA, because of the low signal-to-noise ratio at this concentration. To obtain population-based VIF, additional experiments were performed using DCE-VIBE with the same dose as applied in DCEMOLLI experiment $\left(0.2 \mathrm{mmol} \mathrm{kg}^{-1}\right)$. As an alternative, reference region-based VIF method will be investigated in the future.

Another concern of applying MOLLI sequence to DCE measurements is the change of T1 during dynamic passage of contrast agent. As illustrated by Karlsson and Nordell (2000), the T1 measurements showed good tolerance in normal perfused tissue where the contrast concentration changes linearly during LookLocer T1 mapping. As measured by our DCE method, the contrast agent was infused with a highest wash-in speed around $0.02 \mathrm{mMs}^{-1}$, which is much lower than described in Karlsson 
and Nordell (2000). Thus, under such circumstances, we assumed that the effect of changing T1 during a single MOLLI is negligible.

There exist certain limitations in this study. First, rhabdomyosarcoma (R1) tissue instead of acinar pancreatic carcinoma cell lines (Zhang et al, 2015) was implanted to mimic malignant growths in the pancreas and liver. Despite the significantly different effects shown in our study with respect to the tumour location in relation to therapeutic efficacy, this may not exactly reflect the real responses of the primary and metastatic pancreatic cancers towards the VDA in human patients. Secondly, the sample size of the animals in this experiment appears relatively small, although the intra-individual comparison between pancreatic and hepatic tumours as well as meticulous imaging-histopathologic analyses may make the results more reliable.

To conclude, as shown from this study using multiparametric MRI as an imaging biomarker, both pancreatic head tumours and hepatic tumours presented marked vascular shutdown response early after CA4P intervention. However, because of the unique vascular supply, pancreatic tumours were re-perfused within $10 \mathrm{~h}$ without consequent necrosis, which led to a limited therapeutic response compared with that with hepatic tumours. Hereby, when CA4P is proposed for pancreatic tumour therapy, special attention should be paid to fast tumour re-vascularisation in this organ.

\section{CONFLICT OF INTEREST}

The authors declare no conflict of interest.

\section{REFERENCES}

Chase DM, Chaplin DJ, Monk BJ (2017) The development and use of vascular targeted therapy in ovarian cancer. Gynecol Oncol 145: 393-406.

Chen F, Sun X, De Keyzer F, Yu J, Peeters R, Coudyzer W, Vandecaveye V, Landuyt W, Bosmans H, Van Hecke P, Marchal G, Ni Y (2006) Liver tumor model with implanted rhabdomyosarcoma in rats: MR imaging, microangiography, and histopathologic analysis. Radiology 239: 554-562.

Colliez F, Fruytier AC, Magat J, Neveu MA, Cani PD, Gallez B, Jordan BF (2016) Monitoring Combretastatin A4-induced tumor hypoxia and hemodynamic changes using endogenous MR contrast and DCE-MRI. Magn Reson Med 75: 866-872.

Dark GG, Hill SA, Prise VE, Tozer GM, Pettit GR, Chaplin DJ (1997) Combretastatin A-4, an agent that displays potent and selective toxicity toward tumor vasculature. Cancer Res 57: 1829-1834.

Fruytier A-C, Magat J, Neveu M-A, Karroum O, Bouzin C, Feron O, Jordan B, Cron GO, Gallez B (2014) Dynamic contrast-enhanced MRI in mouse tumors at $11.7 \mathrm{~T}$ : comparison of three contrast agents with different molecular weights to assess the early effects of combretastatin A4. NMR Biomed 27: 1403-1412.

Heisen M, Fan X, Buurman J, Van Riel NAW, Karczmar GS, Ter Haar Romeny BM (2010) The influence of temporal resolution in determining pharmacokinetic parameters from DCE-MRI data. Magn Reson Med 63: 811-816.

Karlsson M, Nordell B (2000) Analysis of the Look-Locker T1 mapping sequence in dynamic contrast uptake studies: simulation and in vivo validation. Magn Reson Imaging 18: 947-954.

Kellman P, Wilson JR, Xue H, Ugander M, Arai AE (2012) Extracellular volume fraction mapping in the myocardium, part 1: evaluation of an automated method. J Cardiovasc Magn Reson 14: 63.

Li J, Chen F, Feng Y, Cona MM, Yu J, Verbruggen A, Zhang J, Oyen R, Ni Y (2013) Diverse responses to vascular disrupting agent combretastatin a4 phosphate: a comparative study in rats with hepatic and subcutaneous tumor allografts using MRI biomarkers, microangiography, and histopathology. Transl Oncol 6: 42-50.
Li J, Sun Z, Zhang J, Shao H, Cona MM, Wang H, Marysael T, Chen F, Prinsen K, Zhou L, Huang D, Nuyts J, Yu J, Meng B, Bormans G, Fang Z, de Witte P, Li Y, Verbruggen A, Wang X, Mortelmans L, Xu K, Marchal G, Ni Y (2011) A dual-targeting anticancer approach: soil and seed principle. Radiology 260: 799-807.

Nathan P, Zweifel M, Padhani AR, Koh DM, Ng M, Collins DJ, Harris A, Carden C, Smythe J, Fisher N, Taylor NJ, Stirling JJ, Lu SP, Leach MO, Rustin GJS, Judson I (2012) Phase I trial of combretastatin A4 phosphate (CA4P) in combination with bevacizumab in patients with advanced cancer. Clin Cancer Res 18: 3428-3439.

Nielsen T, Bentzen L, Pedersen M, Tramm T, Rijken PFJW, Bussink J, Horsman MR, Søtergaard L (2012) Combretastatin A-4 phosphate affects tumor vessel volume and size distribution as assessed using MRI-based vessel size imaging. Clin Cancer Res 18: 6469-6477.

O'Connor JPB, Jackson A, Parker GJM, Roberts C, Jayson GC (2012) Dynamic contrast-enhanced MRI in clinical trials of antivascular therapies. Nat Rev Clin Oncol 9: 167-177.

Okahara M, Mori H, Kiyosue H, Yamada Y, Sagara Y, Matsumoto S (2010) Arterial supply to the pancreas; variations and cross-sectional anatomy. Abdom Imaging 35: 134-142.

Rustin GJ, Shreeves G, Nathan PD, Gaya A, Ganesan TS, Wang D, Boxall J, Poupard L, Chaplin DJ, Stratford MRL, Balkissoon J, Zweifel M (2010) A phase Ib trial of CA4P (combretastatin A-4 phosphate), carboplatin, and paclitaxel in patients with advanced cancer. Br J Cancer 102: 1355-1360.

Salmon HW, Siemann DW (2006) Effect of the second-generation vascular disrupting agent OXI4503 on tumor vascularity. Clin Cancer Res 12: 4090-4094.

Siemann DW, Shi W (2008) Dual targeting of tumor vasculature: combining avastin and vascular disrupting agents (CA4P or OXi4503). Anticancer Res 28: 2027-2031.

Thomas CD, Walczak C, Kaffy J, Pontikis R, Jouanneau J, Volk A (2006) Early effects of combretastatin A4 phosphate assessed by anatomic and carbogen-based functional magnetic resonance imaging on rat bladder tumors implanted in nude mice. Neoplasia 8: 587-595.

Tozer GM, Prise VE, Lewis G, Xie S, Wilson I, Hill SA (2009) Nitric oxide synthase inhibition enhances the tumor vascular-damaging effects of combretastatin A-4 3-O-phosphate at clinically relevant doses. Clin Cancer Res 15: 3781-3790.

Tozer GM, Prise VE, Wilson J, Locke RJ, Vojnovic B, Stratford MRL, Dennis MF, Chaplin DJ (1999) Combretastatin A-4 Phosphate as a Tumor Vascular-Targeting Agent: Early Effects in Tumors and Normal Tissues. Cancer Res 59: 1626-1634.

Wang H, Sun X, Chen F, De Keyzer F, Yu J, Landuyt W, Vandecaveye V, Peeters R, Bosmans H, Hermans R, Marchal G, Ni Y (2009) Treatment of rodent liver tumor with combretastatin a4 phosphate: noninvasive therapeutic evaluation using multiparametric magnetic resonance imaging in correlation with microangiography and histology. Invest Radiol 44: 44-53.

Yin T, Peeters R, Feng Y, Liu Y, Yu J, Dymarkowski S, Himmelreich U, Oyen R, Ni Y (2017a) Characterization of orthotopic pancreatic tumor model using three-dimensional and quantitative multi-parametric MRI. NMR Biomed 30: e3637.

Yin T, Peeters R, Liu Y, Feng Y, Zhang X, Jiang Y, Yu J, Dymarkowski S, Himmelreich U, Oyen R, Ni Y (2017b) Visualization, quantification and characterization of caerulein-induced acute pancreatitis in rats by $3.0 \mathrm{t}$ clinical mri, biochemistry and histomorphology. Theranostics 7: 285-294.

Yushkevich PA, Piven J, Hazlett HC, Smith RG, Ho S, Gee JC, Gerig G (2006) User-guided 3D active contour segmentation of anatomical structures: significantly improved efficiency and reliability. Neuroimage 31: 1116-1128.

Zhang Z, Zheng L, Li W, Gordon aC, Huan Y, Shangguan J, Procissi D, Bentrem DJ, Larson aC (2015) Quantitative functional MRI in a clinical orthotopic model of pancreatic cancer in immunocompetent Lewis rats. Am J Transl Res 7: 1475-1486.

This work is published under the standard license to publish agreement. After 12 months the work will become freely available and the license terms will switch to a Creative Commons AttributionNonCommercial-Share Alike 4.0 Unported License.

Supplementary Information accompanies this paper on British Journal of Cancer website (http://www.nature.com/bjc) 